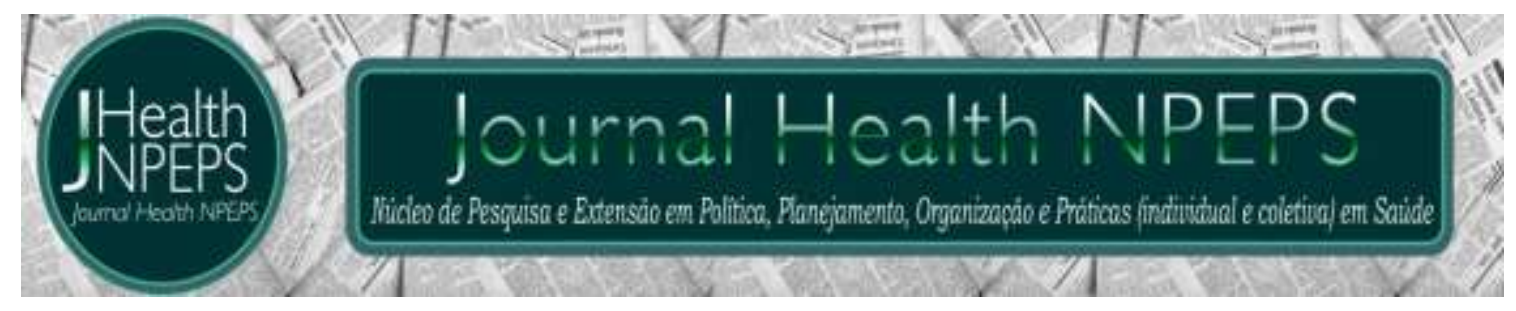

http://dx.doi.org/10.30681/252610103178

ENSAIO TEÓRICO-REFLEXIVO

\title{
O embarque em um aeroporto como exercício de epidemiologia
}

The boarding on an airport as an exercise in epidemiology

El embarque en un aeropuerto como ejercicio de epidemiología

Antônio Augusto Dall'Agnol Modesto ${ }^{1}$

\section{RESUMO}

Objetivo: propor uma narrativa para o ensino de medidas relacionadas a testes diagnósticos e rastreamento através de sua observação no contexto da segurança aeroportuária. Método: trata-se de ensaio teórico-reflexivo, realizado no segundo semestre de 2018, incitado por vivências do autor e experiências na docência universitária. Seus principais referenciais são a epidemiologia clínica, em especial o estudo dos testes diagnósticos, e as narrativas em medicina. Para discutir questões específicas de saúde, foram utilizados artigos de revisão e diretrizes de instituições como o Ministério da Saúde e o Instituto Nacional de Câncer. Resultados: conceitos como sensibilidade, especificidade e valores preditivos positivo e negativo podem ser discutidos através do caso do rastreamento de armas e explosivos no embarque de aeroportos. Considerações finais: situações não relacionadas à saúde dão materialidade a conceitos epidemiológicos fundamentais, facilitando seu ensino-aprendizagem. 0 uso desses exemplos na formação em saúde reforça a interdisciplinaridade buscada nesse campo.

Descritores: Epidemiologia; Técnicas e Procedimentos Diagnósticos; Programas de Rastreamento; Sensibilidade e Especificidade; Pirataria Aérea.

\section{ABSTRACT}

Objective: to propose a narrative for the teaching of measures related to diagnostic tests and screening through their observation in the context of airport security. Method: it is a theoretical-reflexive essay, made in the second half of 2018, prompted by the author's personal experiences and university teaching practices. Its main references are clinical epidemiology, especially the study of diagnostic tests, and narratives in medicine. To discuss specific health issues, review articles and guidelines from institutions such as the Ministry of Health and the National Cancer Institute were used. Results: concepts such as sensitivity, specificity and positive and negative

\footnotetext{
${ }^{1}$ Médico de Família e Comunidade. Doutor em Medicina Preventiva. Docente da Universidade Cidade de São Paulo (Unicid). São Paulo, SP, Brasil. E-mail: aadmodesto@gmail.com ORCID ID: https://orcid.org/0000-0002-5898-3957 Autor principal - Endereço para correspondência: Universidade Cidade de São Paulo - Rua Butantã, 285, Pinheiros São Paulo/SP - CEP 05424-140.
} meio, desde que a publicação original seja corretamente citada. 
predictive values can be discussed through the case of tracking of weapons and explosives during the boarding procedures in airports. Final considerations: nonhealth-related situations give materiality to fundamental epidemiological concepts, facilitating their teaching and learning. The use of such examples in health training reinforces the interdisciplinarity sought in this field.

Descriptors: Epidemiology; Diagnostic Techniques and Procedures; Mass Screening; Sensitivity and Specificity; Hijacking.

\section{RESUMEN}

Objetivo: proponer una narrativa para la enseñanza de medidas relacionadas con pruebas diagnósticas y rastreo a través de su observación en el contexto de la seguridad aeroportuaria. Método: Se trata de un estudio teórico-reflexivo, realizado en el segundo semestre de 2018, incitado por vivencias personales del autor y sus experiencias en la docencia universitaria. Sus referencias son la epidemiología clínica, en especial el estudio de las pruebas diagnósticas, y las narrativas en medicina. Para discutir las preguntas específicas de la búsqueda, artículos de revisión y recomendaciones de instituciones como el Ministerio de Salud y el Instituto Nacional de Cáncer. Resultados: conceptos como sensibilidad, especificidad y valores predictivos positivo y negativo pueden ser discutidos a través del caso del rastreo de armas y explosivos en el embarque de aeropuertos. Consideraciones finales: las situaciones no relacionadas con la salud dan materialidad a conceptos epidemiológicos fundamentales, facilitando su enseñanza-aprendizaje. El uso de dichos ejemplos en la formación en salud refuerza la interdisciplinaridad buscada en ese campo.

Descriptores: Epidemiología; Técnicas y Procedimientos Diagnósticos; Tamizaje Masivo; Sensibilidad y Especificidad; Piratería Aérea.

\section{INTRODUÇÃO}

A Epidemiologia é uma ciência fundamental à Medicina, à Enfermagem e a todas as áreas da saúde, mas é comum que estudantes e profissionais tenham dificuldade em compreendê-la apropriadamente ${ }^{1}$. Com isso, suas habilidades de planejar pesquisas, ler criticamente artigos científicos, avaliar políticas públicas, interpretar exames e escolher tratamentos ficam prejudicadas $^{2,3}$.

Ainda que se fale muito sobre a importância de uma formação interdisciplinar e do uso de metodologias ativas de ensino-aprendizagem na área da saúde ${ }^{4}$, o ensino de testes diagnósticos - tema fundamental em Epidemiologia Clínica - acaba sendo reduzido a uma matriz $2 \times 2$ e uma série de fórmulas e conceitos aplicados a populações feitas de papel e tinta.

Assim, o presente estudo objetivou propor uma narrativa para o ensino de medidas relacionadas a testes diagnósticos e rastreamento através de sua observação no contexto da segurança aeroportuária.

\section{MÉTODO}


Trata-se de um ensaio teóricoreflexivo, realizado no segundo semestre de 2018. A ideia de extrair conceitos de epidemiologia das medidas de segurança implementadas no embarque em um aeroporto surgira no segundo semestre do ano anterior, sendo desde então utilizada satisfatoriamente na universidade, no exercício da docência em sala de aula.

O construto foi elaborado a partir da experiência do próprio autor em aeroportos, tendo em mente as narrativas em Medicina ${ }^{5,6}$, e referenciais da Epidemiologia Clínica, em especial o estudo dos testes diagnósticos ${ }^{2,3,7}$. Para discutir exames de rastreamento específicos, recorreu-se a artigos de revisão e diretrizes de instituições como o Ministério da Saúde (MS) e o Instituto Nacional de Câncer (INCA).

Após um breve ponto de partida, apresenta-se o problema dos sequestros de aviões e, em seguida, são discutidos conceitos como sensibilidade, especificidade, valores preditivos positivo e negativo, através do caso do rastreamento de armas e explosivos no embarque de aeroportos. Dado seu propósito, este ensaio é construído na perspectiva de um momento de ensinoaprendizagem acadêmica.

\section{Ponto de partida}

Há bons textos sobre Epidemiologia Clínica e testes diagnósticos, que podem ser consultados para um ponto de partida mais sólido e nos apoiaram nessa elaboração ${ }^{3,7}$.

Aqui vamos dizer apenas que os testes diagnósticos (como um exame de glicemia ou uma mamografia) têm duas características fundamentais: a confiabilidade e a validade. A confiabilidade é a capacidade de um teste apresentar resultados semelhantes quando aplicado repetidas vezes por examinadores diferentes; a validade é a correspondência de seus achados com a "verdade", e é definida pelas medidas de sensibilidade e especificidade7. A palavra vem entre aspas para lembrar que diagnósticos são definições humanas, históricas e dinâmicas, sujeitas a conflitos de interesse e que precisam ser compreendidas à luz dos fenômenos da medicalização e da promoção da doença (disease mongering $)^{8,9}$. Um exemplo recente é o da deficiência de vitamina $D$, que ganhou ares de pandemia depois que o nível sérico mínimo do hormônio foi aumentado para $30 \mathrm{ng} / \mathrm{mL}$ com base em pesquisas financiadas pela indústria farmacêutica ${ }^{10}$, apesar de uma revisão 
da National Academy of Medicine indicar que $20 \mathrm{ng} / \mathrm{mL}$ de vitamina $D$ dão conta das necessidades de pelo menos $97,5 \%$ dos estadunidenses ${ }^{11}$.

A Tabela 1 é a conhecida matriz 2x2. Ela descreve a aplicação de um teste a uma população da qual se conhecem previamente os indivíduos doentes e não doentes, através de um ou mais exames padrão-ouro (clínicos, anatomopatológicos ou outros) - uma situação de informação total, diferente da que o clínico encontra em seu dia-adia.

Tabela 1- A conhecida matriz $2 \times 2$.

\begin{tabular}{|ccc|}
\hline & Doentes & Sem doença \\
Teste & Verdadeiros & Falsos positivos \\
$\begin{array}{c}\text { positivo } \\
(\mathrm{T}+)\end{array}$ & positivos (VP) & (FP) \\
Teste & Falsos & \\
negativo & negativos & Verdadeiros \\
$(\mathrm{T}-)$ & $(\mathrm{FN})$ & negativos (VN) \\
\hline
\end{tabular}

Quadro 1- Medidas em testes diagnósticos.

\begin{tabular}{l}
\hline Doentes: incluem os resultados VP e FN \\
Sem doença: incluem os resultados VN e FP \\
Sensibilidade: Testes positivos/Doentes \\
Especificidade: Testes negativos/Sem doença \\
Acurácia: VP+VN/População total \\
Valor preditivo positivo: VP/Testes positivos \\
Valor preditivo positivo: VN/Testes negativos \\
FN: Falsos-negativos, FP: Falsos-positivos, VP: \\
Verdadeiros-positivos, VN: Verdadeiros- \\
negativos.
\end{tabular}

\section{O problema dos sequestros de aviões}

A ebulição geopolítica do fim da década de 60 e início da década de 70 foi o contexto para um aumento expressivo nos casos de sequestro de aviões (aircraft hijacking). Em 1972, os Estados Unidos implementaram a detecção de metais no embarque de passageiros, visando impedir que pessoas carregando armas ou explosivos subissem a bordo e realizassem sequestros ou atentados - até então, as pessoas podiam seguir diretamente do balcão da empresa para a aeronave sem serem paradas $^{12}$.

Hoje, os pórticos detectores de metais e os aparelhos de radiografia de bagagem são instrumentos de testes diagnósticos usados em um programa de rastreamento. Em saúde, rastreamento ou rastreio é a busca de doenças ou fatores de risco em pessoas assintomáticas, como realizar sorologias em pessoas que fizeram sexo desprotegido em busca de HIV ou realizar colpocitologia oncótica (o exame de Papanicolaou) periodicamente em mulheres saudáveis, tentando identificar lesões precursoras ou iniciais de câncer de colo uterino ${ }^{13,14}$.

No embarque do aeroporto, os testes diagnósticos buscam identificar pessoas com armas (verdadeiros positivos, VP) dentre todas as pessoas que embarcam (população-alvo do exame). Essas pessoas são “assintomáticas" na medida em que 
estão aparentemente desarmadas. Deixaremos de lado a busca por drogas ou outros itens proibidos, para um recorte mais delimitado e melhor compreensão desse processo; portanto, tenhamos em mente um programa de rastreio que visa evitar sequestros de avião.

É importante lembrar que nem todas as pessoas que embarcam com armas atacarão 0 respectivo voo. Algumas poderão estar traficando a arma; outras, transportando-a com a pretensão de cometer um crime em seu destino. Terroristas embarcados poderão desistir na última hora, e algumas tentativas podem acabar sendo impedidas por alguém a bordo, como uma autoridade à paisana ou um passageiro heroico - uma ação "curativa” quando a prevenção falhou. Assim, uma arma detectada não corresponde diretamente a um sequestro evitado, mas à diminuição brutal do risco de sua ocorrência - ou seja, um passageiro armado é um fator de risco importante para o desfecho de interesse, o sequestro.

0 pórtico detector de metais (PDM) e o aparelho de radiografia de bagagem de mão (ARBM) são instrumentos de alta sensibilidade: 0 detector e a leitura do operador da radiografia identificam boa parte dos objetos que poderiam ser uma arma.

O PDM indica um resultado positivo através de um sinal sonoro. Muitos já viram de perto que isso não significa que 0 passageiro indicado esteja portando uma arma ou explosivo (doravante denominada pessoa armada). Chaves, moedas, fivelas de cinto, cortadores de unha e outros metais inofensivos são capazes de disparar o alarme do pórtico. Sendo assim, esse teste identifica quase todas as pessoas efetivamente armadas (VP), mas também uma quantidade considerável de pessoa portando metais inofensivos (falsos positivos, FP). Tal sensibilidade tranquiliza todos a bordo, pois é mais fácil a máquina errar para mais do que para menos - chatear um passageiro é muito mais provável que liberar um criminoso. Em termos estatísticos, considerando a hipótese nula $\left(\mathrm{H}_{0}\right)$ de que a pessoa rastreada esteja desarmada e a hipótese alternativa dela estar armada $\left(H_{a}\right)$, é mais provável rejeitar a hipótese nula quando ela é verdadeira, ou seja, inferir falsamente a existência de algo ausente (erro tipo I) do que não rejeitar a hipótese nula quando ela é falsa, ou seja, inferir falsamente a ausência de algo que existe (erro tipo II) ${ }^{15}$.

E como são diferenciadas as 
pessoas armadas das desarmadas? Primeiro, sempre que o detector apita, as pessoas são convidadas a checar novamente se não estão portando instrumentos de metal. Esse cuidado aumenta a confiabilidade do teste (pela aplicação da técnica correta, ou seja, desvencilhar-se de itens metálicos) e diminui falsos positivos, pois seu objetivo é identificar armas e explosivos, não metais de qualquer gênero. Isso equivale a pedir a uma pessoa que não fume por 30 minutos antes e descanse sentada por cinco minutos antes de realizar uma medida de pressão arterial, evitando diagnósticos falsos de hipertensão arterial sistêmica.

Se o aparelho apita novamente, as pessoas são submetidas a um ou dois exames mais específicos que os anteriores: a detecção de metais com aparelho portátil (DMAP) e a palpação corporal (PC). Avaliando região por região do corpo, eles são mais capazes de localizar objetos suspeitos e geram pouquíssimos falsos negativos (FN): é difícil ocultar um dispositivo ofensivo de uma palpação corporal bem atenta. Entretanto, eles ainda não são capazes de dar um diagnóstico definitivo sobre o objeto e produzem falsos positivos: um detector portátil pode sinalizar tanto um pequeno revólver preso à cintura (VP) quanto algumas próteses de quadril (FP). Chama-se acurácia a combinação da sensibilidade e da especificidade, ou seja, que parcela de VPs e VNs resultaram em resultados positivos $\mathrm{e}$ negativos, respectivamente (Quadro 1).

Enquanto a pessoa passa pelo pórtico, sua bagagem de mão e pequenos pertences são radiografados. A sensibilidade do teste é a parcela de armas que o agente (e não a máquina) consegue detectar dentre todas as armas e explosivos transitados durante seu turno de trabalho. Assim como o pórtico, a radiografia é um exame de alta sensibilidade: muita gente vai ser parada por causa de secadores de cabelo, mas dificilmente alguém embarcará com uma pistola na maleta. No entanto, sua confiabilidade é provavelmente menor, na medida em que o PDM é um método totalmente automatizado, enquanto as imagens fornecidas pelo ARBM devem ser interpretadas rapidamente por agentes de segurança que recebem treinamento semelhante, mas estão sujeitos à inexperiência ou cansaço. Portanto, trata-se de um exame operadordependente, como a ultrassonografia médica.

O que temos até aqui? Uma primeira etapa, em que dois exames muito sensíveis são aplicados a toda a 
população (passageiros e tripulantes embarcando) e selecionam uma subpopulação de maior risco de portar uma arma, a qual inclui algumas pessoas armadas (VP) e muitas pessoas com objetos inofensivos (FP). Há pouquíssimos falsos negativos (pessoas embarcando armadas). A subpopulação selecionada é submetida a um ou dois exames mais específicos (conforme a positividade dos anteriores), que geram poucos falsos positivos - ou seja, a segunda etapa libera as pessoas incorretamente assinaladas na primeira.

Resultados positivos ao longo das duas etapas exigem um diagnóstico definitivo, realizado através de exames padrão-ouro: a identificação visual e tátil da arma ou explosivo (por vezes exigindo inspeção íntima) e a análise pericial (para confirmar que um bloco de massa seja explosivo plástico, por exemplo). Note-se que o primeiro e mais corriqueiro deles é um exame clínico.

Um exemplo na saúde é o rastreamento de câncer de cólon: a pesquisa de sangue oculto nas fezes é um exame sensível, que seleciona pacientes para um exame mais específico, a colonoscopia, que por sua vez encaminhará alguns indivíduos para - diagnóstico definitivo através do exame anatomopatológico (padrão-ouro) de lesões identificadas, como pólipos ${ }^{13}$. Se já tivermos uma suspeita de câncer de intestino (por perda de peso e constipação intestinal, por exemplo), a pessoa deve ser encaminhada diretamente à colonoscopia, pois não se trata mais de rastreamento, e sim de investigação - a suspeita clínica já colocou o indivíduo em uma subpopulação de risco para a doença.

\section{Valores preditivos}

Uma das perguntas que médicos criteriosos e comprometidos com a prevenção quaternária se fazem ao considerar testes diagnósticos é quanto se pode confiar em um resultado positivo ou negativo. Em síntese, prevenção quaternária são ações visando proteger as pessoas de intervenções diagnósticas e terapêuticas desnecessárias, ou seja, prevenir os excessos da Medicina9,16.

O valor preditivo positivo de um exame é a parcela de verdadeiros positivos dentre os resultados positivos. Se a sensibilidade indica a parcela de pessoas armadas que são identificadas ao passar pelo pórtico, o VPP indica a parcela de pessoas efetivamente armadas dentre aquelas para as quais o pórtico apitou. Da mesma forma, o valor preditivo negativo equivale à parcela de 
verdadeiros negativos dentre os resultados negativos. Enquanto a especificidade do pórtico é a parcela de pessoas desarmadas que ele deixa passar, o VPN é a parcela de pessoas efetivamente desarmadas dentre as que não disparam o alarme.

VPP e VPN não são
características próprias do teste
diagnóstico, mas sim resultado de sua
aplicação em uma população específica.
Eles dependem da prevalência da condição buscada na população de estudo, baseada nos registros disponíveis.

Voltemos ao aeroporto: um PDM apita para um passageiro. Os agentes de segurança teriam a mesma preocupação se ele fosse uma criança ou um homem adulto? Provavelmente não, considerando que mais homens levem armas a bordo do que crianças. Se tivessem que escolher entre revistar apenas a criança ou o homem adulto e escolhessem a primeira por pressentimento, estariam contrariando registros objetivos e incorrendo em um viés do examinador.

A alta sensibilidade do teste e a baixíssima prevalência de pessoas tentando embarcar armadas fazem com que o PDM seja um teste com baixo valor preditivo positivo. Ainda assim, ele seleciona uma população com a prevalência um pouco maior de pessoas armadas, aumentando o valor preditivo positivo dos testes seguintes.

No caso da saúde, os dados de prevalência advêm principalmente das informações epidemiológicas locais e de pesquisas exploratórias em contextos semelhantes. A experiência do profissional é um fator importante, mas deve ser usada com cuidado. Muitos médicos que testemunham uma doença grave em uma pessoa fora da idade mais comum de ocorrência (como câncer de mama em uma mulher de 30 anos) passam a solicitar exames de rastreamento excessivos ou para mais pessoas do que o recomendado - por exemplo, ultrassonografias de mama para mulheres assintomáticas de 25 anos. Aplicados a uma população com menor prevalência da doença, esses testes acabam gerando muitos falsos positivos e intervenções desnecessárias ${ }^{9,17}$.

A relação entre valor preditivo positivo e prevalência foi formulada pelo matemático Thomas Bayes (c. 17011761) e publicada dois anos após sua morte. O Teorema de Bayes descreve a relação entre probabilidades condicionadas, ou como a probabilidade pós-teste (equivalente ao VPP) varia 
conforme a probabilidade pré-teste $(\mathrm{a}$ probabilidade de estar doente, ou seja, a prevalência da doença na população estudada) ${ }^{18}$.

\section{Por que não começar com a segunda etapa?}

Se o detector de metais portátil e a palpação corporal são exames mais específicos, por que não os realizar em todos os passageiros? Porque seria custoso (pela necessidade de mais agentes trabalhando simultaneamente) e inconveniente (pela demora e invasão de privacidade) a ponto de impedir sua aplicação universal, sendo preferíveis para tanto o PDM e o ARBM.

A Organização Mundial de Saúde (OMS) definiu critérios para o estabelecimento de programas de rastreamento ${ }^{19}$ que podem ser aplicados tanto à colonoscopia para rastreio de câncer quanto à palpação corporal para detecção de armas e explosivos.

Sobre o item 10, o esquema de segurança só funciona porque é universal e continuado. Homens, mulheres, idosos, crianças, pessoas com deficiência, membros da tripulação, todos são submetidos ao rastreamento de armas no embarque de todas as aeronaves comerciais - e não apenas as mulheres em outubro e os homens em novembro, por exemplo ${ }^{20}$. No caso dos rastreamentos em saúde, esse critério valida a necessidade de um sistema com acesso universal e abordagem integral, com uma Atenção Primária forte ${ }^{21}$.

Quadro 2 - Critérios para o estabelecimento de programas de rastreamento.

1. A condição procurada deve ser um problema de saúde importante.

2. Deve haver um tratamento aceitável para pacientes com a doença reconhecida.

3. Dispositivos de diagnóstico e tratamento devem estar disponíveis.

4. Deve haver um estágio latente ou oligossintomático reconhecível.

5. Deve haver um teste ou exame físico adequado.

6. O teste deve ser aceito pela população

7. A história natural da condição, incluindo a evolução da doença latente à declarada, deve ser adequadamente compreendida.

8. Deve haver uma política consensuada sobre quem tratar como pacientes.

9. 0 custo da busca ativa (incluindo diagnóstico e tratamento de pacientes diagnosticados) deve ser economicamente balanceado em relação ao gasto possível com cuidado médico em geral.

10. A busca ativa deve ser um processo contínuo, e não um projeto pontual.

Fonte: Adaptado de Wilson e Jungner ${ }^{19}$.

\section{CONSIDERAÇÕES FINAIS}

O ensino em saúde deve romper as grades dos leitos e as paredes da universidade se pretende formar profissionais com compreensão integral do processo saúde-doença. Nesse ensaio, foram discutidos conceitos epidemiológicos tomando como exemplo algumas medidas de segurança no embarque de aeroportos. Situações não relacionadas à saúde dão materialidade 
a conceitos epidemiológicos fundamentais, facilitando seu ensinoaprendizagem. E seu uso na formação em saúde reforça a interdisciplinaridade buscada nesse campo, onde não se precisa diferenciar criminosos de inocentes, mas doença do que parece doença.

As limitações desse estudo relacionam-se ao seu caráter ensaístico e sua revisão bibliográfica não sistematizada. Nossa expectativa é inspirar elaborações interdisciplinares semelhantes, que podem dar conta de outras questões de difícil aprendizado, como a bioestatística.

\section{REFERÊNCIAS}

1. Keyes KM, Galea S. Current Practices in Teaching Introductory Epidemiology: How We Got Here, Where to Go. Am J Epidemiol. 2014; 180(7):661-8.

2. Stein AT. Medicina Baseada em Evidências aplicada à prática do médico de família. In: Gusso G, Lopes JMC. Tratado de Medicina de Família. Porto Alegre: Artmed; 2012.

3. Lotufo PA, Benseñor IM, Olmos RD. Epidemiologia clínica. In: Gusso G, Lopes JMC. Tratado de Medicina de Família e Comunidade: princípios, formação e prática. Porto Alegre: Artmed; 2012.

4. Mitre SM, Siqueira-Batista R, Girardide-Mendonça JM, Morais-Pinto NM, Meirelles $C A B$, Pinto-Porto $C$, et al. Metodologias ativas de ensinoaprendizagem na formação profissional em saúde: debates atuais. Ciênc saúde coletiva. 2008; 13(Sup 2):2133-44

5. Favoreto CAO, Camargo Júnior KR. A narrativa como ferramenta para o desenvolvimento da prática clínica. Interface (Botucatu). 2011; 15(37):473-83.

6. Grossman E, Cardoso MHCA. As narrativas em medicina: contribuições à prática clínica e ao ensino médico. Rev Bras Educ Med. 2006; 30(1):6-14.

7. Almeida Filho ND, Rouquayrol MZ. Diagnóstico em epidemiologia. In: Almeida Filho ND, Rouquayrol MZ. Introdução à Epidemiologia. Rio de Janeiro: Guanabara Koogan; 2013.

8. Moynihan R, Heath I, Henry D. Selling sickness: the pharmaceutical industry and disease mongering. BMJ. 2002; 324:886-91.

9. Norman $A H$, Tesser CD. Prevenção quaternária na atenção primária à saúde: uma necessidade do Sistema Único de Saúde. Cad Saúde Pública. 
2009; 25(9):2012-20.

10. Szabo L. Vitamin D, the Sunshine Supplement, Has Shadowy Money Behind It. The New York Times [online]. 18 ago 2018. Disponível em https://www.nytimes.com/2018/08/ 18/business/vitamin-d-michaelholick.html. Acesso em 20 out 2018.

11. Ross AC, Manson JE, Abrams SA, et al. The 2011 Report on Dietary Reference Intakes for Calcium and Vitamin D from the Institute of Medicine: What Clinicians Need to Know. J Clin Endocrinol Metab. 2011; 96(1):53-8.

12. Wu A. The History of Airport Security The Savvy Traveler [online]. 2004. Disponível em http: / / savvytraveler.publicradio.org /show/features/2000/20000915/sec urity.shtml. Acesso em 9 set 18.

13. Ministério da Saúde (BR). Secretaria de Atenção à Saúde. Cadernos de Atenção Básica - n 29. Departamento de Atenção Básica. Rastreamento. Brasília: Ministério da Saúde; 2010.

14. Norman AH, Tesser CD. Rastreamento de doenças. In: Gusso G, Lopes JMC (orgs). Tratado de Medicina de Família e Comunidade: princípios, formação e prática. Porto Alegre: Artmed; 2012.

15. Costa SF. Introdução ilustrada à
Estatística. São Paulo: Harbra; 2013.

16. Jamoulle M, Gusso G. Prevenção quaternária: primeiro não causar dano. In: Gusso G, Lopes JMC. Tratado de Medicina de Família e Comunidade: princípios, formação e prática. Porto Alegre: Artmed; 2012.

17. Migowski A, Azevedo e Silva G, Dias MBK, et al. Diretrizes para detecção precoce do câncer de mama no Brasil. II - Novas recomendações nacionais, principais evidências e controvérsias. Cad Saúde Pública. 2018; 34(6):e00074817.

18. Routledge R. Bayes's Theorem. In: Encyclopædia Britannica [online]. Encyclopædia Britannica, inc.; $7 \mathrm{fev}$ 2018. Disponível em https://www.britannica.com/topic/ Bayess-theorem. Acesso em 20 out 2018.

19. Wilson JMG, Jungner G. Principles and practice of screening for disease. Genebra: World Health Organization; 1968.

20. Modesto AAD, Lima RLB, D’Angelis AC, Augusto DK. Um novembro não tão azul: debatendo rastreamento de câncer de próstata e saúde do homem. Interface (Botucatu). 2018; 22(64):251-62.

21. Giovanella L, Mendonça MHM. Atenção Primária à Saúde. In: 
Giovanella L, Escorel S, Lobato LVC, Rio de Janeiro: Editora Fiocruz; Noronha JC, Carvalho Al. Políticas e 2012.

Sistema de Saúde no Brasil. 2a ed.

Conflito de interesses: Os autores declaram não haver conflito de interesses.

Participação dos autores:

- Concepção: Modesto AAD.

- Desenvolvimento: Modesto AAD.

- Redação e revisão: Modesto AAD.

Como citar este artigo: Modesto AAD. 0 embarque em um aeroporto como exercício de epidemiologia. J Health NPEPS. 2019; 4(1):341-352.

Submissão: 25/01/2019

Aceito: $20 / 05 / 2019$

Publicado: 01/06/2019 